\title{
A new ensemble algorithm of differential evolution and backtracking search optimization algorithm with adaptive control parameter for function optimization
}

\author{
Sukanta Nama ${ }^{a^{*}}$, Apu Kumar Saha ${ }^{a}$ and Sima Ghosh ${ }^{b}$
}

${ }^{a}$ Department of Mathematics, National Institute of Technology Agartala, Jirania, Agartala, Tripura-799046, India

${ }^{b}$ Department of Civil Engineering, National Institute of Technology Agartala, Jirania, Agartala, Tripura-799046, India

\begin{tabular}{l}
\hline C H R O N I C L E \\
\hline Article history: \\
Received July 212015 \\
Received in Revised Format \\
August 162015 \\
Accepted September 82015 \\
Available online \\
September 12 2015 \\
\hline Keywords: \\
Differential Evolution (DE) \\
Backtracking Search Optimization \\
Algorithm (BSA) \\
Ensemble Algorithm \\
Unconstrained Optimization
\end{tabular}
A B S T R A C T

Differential evolution (DE) is an effective and powerful approach and it has been widely used in different environments. However, the performance of DE is sensitive to the choice of control parameters. Thus, to obtain optimal performance, time-consuming parameter tuning is necessary. Backtracking Search Optimization Algorithm (BSA) is a new evolutionary algorithm (EA) for solving real-valued numerical optimization problems. An ensemble algorithm called E-BSADE is proposed which incorporates concepts from DE and BSA. The performance of E-BSADE is evaluated on several benchmark functions and is compared with basic DE, BSA and conventional DE mutation strategy. Also the performance results are compared with state of the art PSO variant.

\section{Introduction}

In finding the solution of non-linear problem, the optimization algorithm is most vital and durable. Since some of the problem has discontinuous, gradient based algorithms do not seem to be used for that sort of problems, moreover, gradient search in issues with greater than one local optimum is tough and unstable. Optimization Technique is an effective and powerful technique for that issues. A vast amount of research has been developed on this topic some of them are Particle Swarm Optimization (Kennedy \& Eberhart, 1995), Differential Evolution (Storn \& Price, 1995), Harmony Search Algorithm (Lee \& Geem, 2005), Tabu Search (Glover \& Laguna, 1997), Ant Colony Optimization (Blum, 2005), Teaching Learning Based Optimization (Rao et al., 2011; Rao \& Patel, 2012, 2013), Water Cycle Algorithm (Eskandar et al., 2012), Mine Blast Algorithm (Sadollaha et al., 2013), Backtracking Search Optimization (Civicioglu, 2013). In recent years, it has become evident that the concentration on a sole meta-heuristic is rather restrictive. A skilled combination of meta-heuristic with other optimization techniques, so called hybrid meta-heuristic, can provide a more efficient behavior and a higher flexibility when dealing with real- 
world and large scale problems. For the above reasons, the growing interest in this field of hybrid metaheuristics and some of its latest applications to a wide range of problems can be summarized in references (Zolfaghari et al., 2005; Zhang et al., 2007; Fan \& Zahara, 2007; Nourelfath et al., 2007; Kao \& Zahara, 2008; Sengupta \& Upadhyay, 2009; Behnamian et al., 2009; Gong et al., 2010.; Liu et al., 2010; Lin, 2010; Rao \& Savsani 2012; Shojaeefard et al., 2013; Das, 2014; Guo et al., 2014; Nama et al., 2015; Parouha \& Das, 2015) for the last decade.

Backtracking search optimization algorithm (BSA) is a new evolutionary algorithm (EA) which was proposed by Pinar Civicioglu (2013). BSA uses random mutation strategy with one direction individual for each target individual and a non-uniform crossover strategy which is different and more complex than the crossover strategies used in many genetic algorithms. The DE is also a population-based heuristic algorithm, and it has two control parameters; one is weighting coefficient and the other is crossover probability; parameter weighting coefficient $(\mathrm{F})$ is used to generate new trial solutions; the crossover probability (CR) is used to determine how much of a trial solution should be adopted into the current solution, as well as the DE scheme which is the crucial idea behind DE for generating trial vectors. Recently Suganthana (Mallipeddia et al., 2011) proposed an ensemble of mutation strategies and parameter values for DE (EPSDE) in which a pool of mutation strategies, along with a pool of values corresponding to each associated parameter competes. In this paper, we propose an ensemble of DE and BSA (called E-DEBSA) which provides the more effectiveness from the other algorithms. The remainder of this paper is organized as follows: a brief overview of DE and BSA is presented in Section 2; Section 3 describes the proposed algorithm. The experimental result using different test functions is described in Section 4. Finally, Section 5 gives a brief conclusion about this study.

\section{Overview of DE and BSA}

In this section about the basic concept of DE and BSA are discussed.

\subsection{Basics of $D E$}

Differential evolution, a stochastic search evolutionary algorithm which is based on the Darwin theory of evaluation. The advantage of this algorithm is that it not only uses a few control variables, but also performs well in convergence. DE is introduced to solve the global optimization by Storn and Price (1995). At generation zero the initial population should better cover the entire search space as much as possible by uniformly randomizing individuals within the search space. A candidate replaces the individual only if it is better than its individual. Thereafter, DE guides the population towards the vicinity of the global optimum through repeated cycles of mutation, crossover and selection. The main procedure of DE is explained in detail as follows.

\subsubsection{Mutation}

After initialization, DE employs the mutation operation to produce a mutant vector $v_{i, j}$ with respect to each individual $X_{i, j}$ so-called target vector, in the current population. For each target vector $X_{i, j}$ at the generation $j$, its associated mutant vector $V_{i, j}$ can be generated via certain mutation strategy. The following are different mutation strategies frequently used in the literature:

$$
\begin{array}{ll}
\text { "DE/rand/1": } & v_{i, j}=X_{i, j}^{r_{1}}+F *\left(X_{j}^{r_{2}}-X_{j}^{r_{3}}\right) \\
\text { "DE/current-to-rand/1": } & v_{i, j}=X_{j}^{r_{1}}+K *\left(X_{j}^{r_{1}}-X_{j}^{r_{3}}\right)+F *\left(X_{j}^{r_{4}}-X_{j}^{r_{5}}\right) \\
\text { "DE/best/2": } & v_{i, j}=X_{j}^{\text {best }}+F *\left(X_{j}^{r_{1}}-X_{j}^{r_{2}}\right)+F *\left(X_{j}^{r_{3}}-X_{j}^{r_{4}}\right) \\
\text { "DE/rand-to-best/1" or "DE/target-to-best/1": } \\
v_{i, j}=X_{j}^{r_{1}}+K *\left(X_{j}^{\text {best }}-X_{j}^{r_{3}}\right)+F *\left(X_{j}^{r_{4}}-X_{j}^{r_{5}}\right)
\end{array}
$$


The indices $r_{1}, r_{2}, r_{3}, r_{4}, r_{5}$ are mutually exclusive integers randomly chosen from the range [1, NP]. These indices are randomly generated once for each mutant vector. The weighting coefficient $F$ is a positive control parameter for scaling the difference vector. $X_{j}^{\text {best }}$ is the best fitness individual vector in the population. $K$ is randomly chosen within the range $[0,1]$. In this paper $F$ is chosen as 0.5 .

\subsubsection{Crossover operation}

After the mutation phase, DE binomial crossover operation is applied to $X_{i}$ (target vector) and its corresponding $v_{i, j}$ (mutant vector) to generate a trial vector:

$u_{i, j}=\left(u_{i, 1}, u_{i, 2}, u_{i, 3}, \ldots \ldots \ldots, u_{i, D}\right)$

$$
u_{i, j}=\left\{\begin{array}{lr}
v_{i, j} & \text { if } \operatorname{rand}_{j}(0,1)<C R \\
X_{i, j} & \text { Otherwise }
\end{array}\right.
$$

where $i=1,2,3, \ldots \ldots \ldots, N P ; j=1,2,3, \ldots \ldots \ldots, D$. In Eq. (5), the crossover rate CR controls the fraction of parameter values copied from the mutant vector. The crossover rate is chosen as 0.9 . If the trial vector $u_{i, j}$ violets the boundary condition, it is reset as follows:

$$
u_{i, j}=\left\{\begin{array}{c}
\mathrm{ll}_{\mathrm{i}}+\operatorname{rand}(0,1) *\left(\mathrm{ul}_{\mathrm{i}}-\mathrm{ll}_{\mathrm{i}}\right) \\
\mathrm{ul}_{\mathrm{i}}-\operatorname{rand}(0,1) *\left(\mathrm{ul}_{\mathrm{i}}-\mathrm{ll}_{\mathrm{i}}\right) \quad \text { if } u_{i, j}<\mathrm{ll}_{\mathrm{i}} \\
u_{i, j}>\mathrm{ul}_{\mathrm{i}}
\end{array}\right.
$$

where $i=1,2,3, \ldots \ldots \ldots ., N P ; j=1,2,3, \ldots \ldots \ldots, D, 1_{\mathrm{i}}$ represents the lower limit and uli represents the upper limit in the search space of the ith population. The selection operator is performed to select the better one from the target vector $X_{i}$ and the trial vector $u_{i, j}$ to enter the next generation by Eq. (7).

$$
X_{i, j}=\left\{\begin{array}{lr}
u_{i, j} & \text { if } f\left(u_{i, j}\right)<f\left(X_{i, j}\right) \\
X_{i, j} & \text { Otherwise }
\end{array}\right.
$$

The Algorithm step of basic DE is as follows,

Step 1. Generalized initial population, algorithm parameter;

Step 2. Evaluate the fitness of each individual in the population;

Step 3. Generate the mutant vector for each target vector mutation strategy;

Step 4. By crossover operator generate trial vector for each target vector which are given in Eq.(5);

Step 5. Evaluate fitness vector of each target vector;

Step 6. Select individual between the target (parent) and trial vector using Eq. (7);

Step 7. If the stopping criterion is not satisfied go to Step 3, else return the individual with the best

fitness as the solution.

\subsection{Basics of $B S A$}

BSA, a population-based iterative EA, not only possess the advantage of using few control variables but also performs well in convergence. BSA is introduced to solve the global optimization by Pinar Civicioglu. At the initial stage the initial population uniformly and randomly generated within the search space. BSA determines the historical population oldP for calculating the search direction. The initial historical population is determined within the search boundary. BSA has the option of redefining oldP at the beginning of each iteration through the Eq. (1):

$$
\text { if } \mathrm{a}<\mathrm{b} \text { then, } \operatorname{OldP}=\mathrm{P}, \quad \text { where }(\mathrm{a}, \mathrm{b}) \in \operatorname{rand}(0,1)
$$

Eq. (8) ensures that BSA designates a population belonging to a randomly selected previous generation as the historical population and remembers this historical population until it is changed. Thus, BSA has a memory. After oldP is determined, Eq. (9) is used to randomly change the order of the individuals in oldP: 


\subsubsection{Mutation}

During each generation, BSA's mutation process generates the initial form of the trial population Mutant using Eq. (10) as follows,

$$
\text { Mutant }=P+F \times(\text { old } P-P) \text {. }
$$

Here $F$ controls the amplitude of the search-direction matrix (oldP - P). As the historical population is employed within the calculation of the search-direction matrix, BSA generates a trial population, taking partial advantage of its experiences from previous generations. The control parameter is $\mathrm{F}=3 \times \mathrm{rndn}$, where rndn $\epsilon \mathrm{N}(0,1)$.

\subsubsection{Crossover}

After the new mutant operation is finished, the crossover process generates the final form of the trial population $T$. The initial value of the trial population is Mutant, which has been set in the mutation process. Individuals with better fitness values for the optimization problem are used to evolve the target population. The first step of the crossover process calculates a binary integer-valued matrix $(\mathrm{H})$ of size $N \times D$ that indicates the individuals of $T$ to be manipulated using the relevant individuals of $P$. Then, the trial population $T$ is updated as given in Algorithm 1. In Algorithm 1 two predefined strategies are randomly used in defining the integer-valued matrix, which is more complex than the processes used in DE. The first strategy uses mix rate $\mathrm{M}$, and the other allows only one randomly chosen individual to mutate in each trial. The Algorithm step of basic BSA is as follows,

Step 1. Generalized initial population, algorithm parameter;

Step.2 Evaluate the fitness of each individual in the population;

Step 3. Generate mutant population by Eq. (10)

Step 4. By using Crossover Strategy given in Algorithm 1, generates the trial population.

Step 5. Evaluate fitness vector of the trial population (offspring)

Step 6. Select individual between target population and trial population (offspring)

Step 7. Update individual

Step 8. If the stopping criterion is not satisfied go to Step 3, else return the individual with the best fitness as the solution.

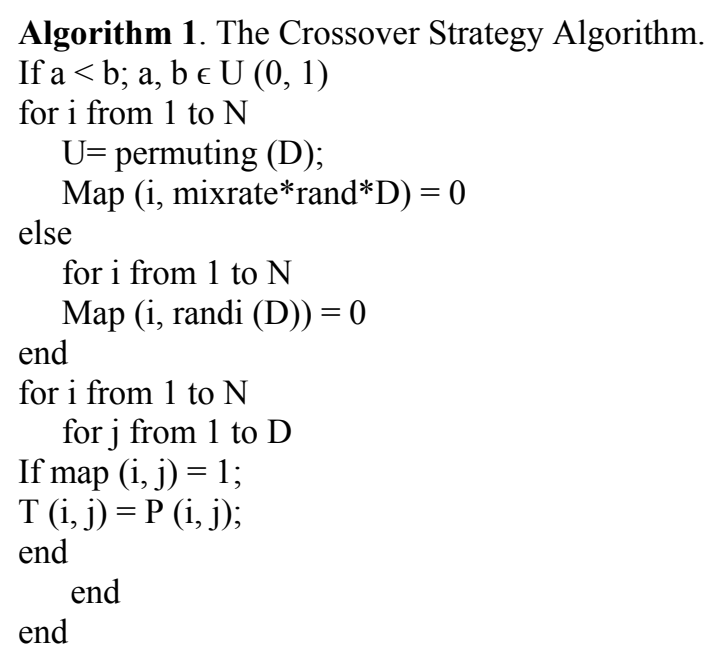

\section{Proposed method}

The combination of meta-heuristic with other optimization techniques is called hybrid meta-heuristic which can provide a more efficient behavior and a higher flexibility when dealing with real-world and large scale problems. Recently, many researchers developed the hybrid meta-heuristic optimization 
technique and used for optimization. Recently Mallipeddia et al. (2011) proposed an ensemble of mutation strategies and parameter values for DE called (EPSDE) in which a pool of mutation strategies, along with a pool of values corresponding to each associated parameter competes. In this paper, we proposed an ensemble of DE and BSA (E-DEBSA) which is more effectiveness compared with the others algorithm. In the proposed approach, the algorithm initialized with a population of opposition based (Tizhoosh, 2005; Rahnamayan et al., 2008) random solutions and searches near the optima by traveling into the search space. During this travel an evolution of this solution is performed by ensemble DE and BSA. The detail described of the proposed method is as follows.

\subsection{Crossover rate (CR)}

The crossover rate that is used to determine how much of a trial solution should be adopted into the current solution, as well as the DE scheme which is the crucial idea behind DE for generating trial vectors (Zaharie, 2009). The crossover rate $\mathrm{CR}$ is a probability $(0 \leq \mathrm{CR} \leq 1)$ of mixing between trial and target vectors. A large CR speeds up convergence (Storn, 1996; Gämperle et al., 2002). According to Storn and Price (1997), $\mathrm{CR}=0.1$ is a good initial choice while $\mathrm{CR}=0.9$ or 1.0 can be tried to increase the convergence speed. According to Gämperle et al. (2002), a good choice for CR is said to be between 0.3 and 0.9. When $\mathrm{CR}=1.0$ is chosen, the number of trial solutions will be reduced dramatically which may lead to stagnation (Lampinen \& Zelinka, 2000; Rönkkönen et al., 2005). Therefore, $C R=0.9$ or 0.99 can be used instead. Varying the values of CR during the course of a run can improve its performance (Smuc, 2002). The modified Crossover rate (CR) is defined as

$$
C R=C R_{\min }+\left(C R_{\max }-C R_{\min }\right) \frac{f^{i}{ }_{\text {max }}-f^{i}{ }_{\text {min }}}{f^{0}{ }_{\text {max }}-f^{0}{ }_{\text {min }}},
$$

where $\mathrm{CR}_{\max }=0.9$ and $\mathrm{CR}_{\min }=0.3 ; f^{0}{ }_{\max }$ and $f^{0}{ }_{\text {min }}$ are the maximum and minimum initial function value; $f^{i}{ }_{\max }$ and $f^{i}{ }_{\text {min }}$ are the maximum and minimum function value at iteration $i$, respectively.

\subsection{Scaling factor/ weighting coefficient (F)}

Scaling factor, $F$ that is used to generate new trial solutions and is usually chosen between 0.5 and 1 (Storn 1996). Storn and Price (1997) found that values of Scaling factor $(F)$ smaller than 0.4 and greater than 1.0 are occasionally effective. Gämperle et al. (2002) and Rönkkönen et al. (2005) also justified that a large value of $F$ increases the probability of escaping from a local optimum. When $F$ is greater than one, the number of function evaluations to find the optimum grows very quickly and convergence speed decreases making it difficult to converge as the perturbation is larger than the distance between two members. $\mathrm{F} \leq 1$ is usually both faster and reliable. Storn and Price (1997) and Gämperle et al. (2002) said that $F=0.6$ or 0.5 would be a good initial choice while Rönkkönen et al. (2005) reported that $F=0.9$ would be a good initial choice. Typical values of $\mathrm{F}$ are $0.4-0.95$ according to Rönkkönen et al. (2005). Smuc (2002) said that varying the values of $F$ during the course of a run can improve its performance. So the modified Scaling factor $(\mathrm{F})$ is defined as

$$
F=F_{\text {min }}+\left(F_{\text {max }}-F_{\text {min }}\right) \frac{f^{i}{ }_{\text {max }}-f^{i}{ }_{\text {min }}}{f^{0}{ }_{\text {max }}-f^{0}{ }_{\text {min }}},
$$

where $\mathrm{F}_{\max }=0.95$ and $\mathrm{F}_{\min }=0.4 ; f^{0}{ }_{\max }$ and $f^{0}{ }_{\text {min }}$ are the maximum and minimum initial function values; $f^{i}{ }_{\max }$ and $f^{i}{ }_{\min }$ are the maximum and minimum function value at iteration $i$, respectively.

\subsection{BSA Control parameter}

Another modification is related to the BSA Control parameter $(F)$ of the basic BSA algorithm. BSA control parameter $(F)$ controls the amplitude of the search-direction matrix (oldP - P). In the basic BSA, the control parameter $(F)$ was chosen as $3 \times \operatorname{rndn}$, where $\operatorname{rndn} \sim N(0,1)(N$ is the standard normal distribution). Thus during the course of optimization the objective function updates only with the better choice of the control parameter. Considering this fact, BSA Control parameter $(F)$ is modified in the present work. This modification increases the exploration and exploitation capacity of the proposed 
algorithm. In the optimization algorithm, the lower value of BSA control parameter (F) allows the fine search in small steps but causes low convergence. A larger value of BSA Control parameter (F) speeds up the search but it reduces the exploration capability. The modified BSA control parameter $(\mathrm{F})$ is defined as

$$
F=1-\frac{f^{i}{ }_{\text {max }}-f^{i}{ }_{\text {min }}}{f^{0}{ }_{\text {max }}-f^{0}{ }_{\text {min }}} \operatorname{rand}(0,1),
$$

where $f^{0}{ }_{\text {max }}$ and $f^{0}{ }_{\text {min }}$ are the maximum and minimum initial function value; $f^{i}{ }_{\max }$ and $f^{i}{ }_{\min }$ are the maximum and minimum function value at iteration $i$, respectively. Thus in the proposed hybrid algorithm the control parameter (F) varies automatically during the search. Control parameter improves the performance of the hybrid algorithm. Implementation steps of the proposed hybrid algorithm is summarized below

Step 1. Initialization: Initialize a population randomly and oppositional based population (OP) on ndimensions in the problem space. Evaluate the fitness value. Select NP number of fittest vectors from $\{\mathrm{P}, \mathrm{OP}\}$ as initial population set,

Step 2. DE Mutation: Calculate the population, according to Eq. (1),

Step 3. DE Crossover: Crossover is an operator to generate new strings (i.e., offspring) from parent strings,

Step 4. Updating the population and fitness vector:

Step 5. BSA Mutation: Calculate the population, according to Eq. (10),

Step 6. BSA Crossover: Crossover is an operator to generate new strings (i.e., offspring) from parent strings,

Step 7. Updating the population and fitness vector:

Step 8. Based on a jumping rate $\mathrm{Jr}$ (i.e. Jumping probability), OP is generated and fitness value of the OP is calculated,

Step 9: Select require number of fittest individuals from $\{\mathrm{P}, \mathrm{OP}\}$ as current Organisms,

Step 10: If the stopping criterion is not satisfied go to Step 2, else return the individual with the best fitness as the solution.

\section{Experimental study and Discussion}

21 different benchmark functions are taken for verification of the proposed algorithm which is given in Appendix 1. In our experimental studies, the performance of the algorithms, the average and standard deviation of the error function value $\left(\mathrm{f}(\mathrm{x})-\mathrm{f}\left(\mathrm{x}^{*}\right)\right)$ were recorded (Suganthan et al., 2005) for measuring the performance of each algorithm, here $x$ is the best solution found by the algorithm in a run and $x^{*}$ is the global optimum of the test function. The Number of successful runs (SR) and successful performance (SP) are recorded before reaching the VTR.

\section{Table 1}

Comparative statistical results of 13 benchmark function of E-BSADE for different values of population size (PS) at $\mathrm{D}=30$ over 25 runs, after 200000 function evaluations.

\begin{tabular}{|c|c|c|c|c|c|}
\hline Function & & $\mathrm{PS}=20$ & $\mathrm{PS}=50$ & $\mathrm{PS}=70$ & $\mathrm{PS}=100$ \\
\hline F1 & Mean(Std.) & $6.08 \mathrm{e}-151(3.04 \mathrm{e}-150)$ & $1.25 \mathrm{e}-113(5.36 \mathrm{e}-113)$ & $3.33 \mathrm{e}-075(7.74 \mathrm{e}-075)$ & $3.41 \mathrm{e}-047(9.84 \mathrm{e}-047)$ \\
\hline F2 & Mean(Std.) & $4.47 \mathrm{e}-010(2.22 \mathrm{e}-009)$ & $4.25 e-059(9.78 e-059)$ & $3.23 \mathrm{e}-039(5.82 \mathrm{e}-039)$ & $1.16 \mathrm{e}-025(1.82 \mathrm{e}-025)$ \\
\hline F3 & Mean(Std.) & $7.42 \mathrm{e}-016(3.71 \mathrm{e}-015)$ & $1.43 e-100(7.13 e-100)$ & $3.99 \mathrm{e}-066(1.52 \mathrm{e}-065)$ & $8.22 \mathrm{e}-042(2.16 \mathrm{e}-041)$ \\
\hline F5 & Mean(Std.) & $3.48 \mathrm{e}+001(2.36 \mathrm{e}+001)$ & $2.79 \mathrm{e}+001(2.96 \mathrm{e}-001)$ & $2.77 \mathrm{e}+001(3.96 \mathrm{e}-001)$ & $2.74 \mathrm{e}+001(3.50 \mathrm{e}-001)$ \\
\hline F6 & Mean(Std.) & $0.00 e^{+000}\left(0.00 e^{+000}\right)$ & $0.00 \mathrm{e}+000(0.00 \mathrm{e}+000)$ & $0.00 \mathrm{e}+000(0.00 \mathrm{e}+000)$ & $0.00 e^{-000}\left(0.00 e^{+000}\right)$ \\
\hline F7 & Mean(Std.) & $2.95 e-004(1.37 e-004)$ & $6.72 \mathrm{e}-004(3.25 \mathrm{e}-004)$ & $1.27 \mathrm{e}-003(5.66 \mathrm{e}-004)$ & $1.60 \mathrm{e}-003(5.87 \mathrm{e}-004)$ \\
\hline F8 & Mean(Std.) & $2.45 \mathrm{e}-001(1.47 \mathrm{e}-001)$ & $2.46 \mathrm{e}-001(2.20 \mathrm{e}-001)$ & $2.15 e-001(1.55 e-001)$ & $3.12 \mathrm{e}-001(2.94 \mathrm{e}-001)$ \\
\hline F9 & Mean(Std.) & $7.27 e+000(1.37 e+001)$ & $5.20 \mathrm{e}-001(1.54 \mathrm{e}+000)$ & $1.33 e+000(2.89 e+000)$ & $0.00 \mathrm{e}+000(0.00 \mathrm{e}+000)$ \\
\hline F10 & Mean(Std.) & $8.05 \mathrm{e}-002(4.03 \mathrm{e}-001)$ & $1.46 \mathrm{e}-015(1.33 \mathrm{e}-015)$ & $2.45 \mathrm{e}-015(1.80 \mathrm{e}-015)$ & $2.03 \mathrm{e}-015(1.69 \mathrm{e}-015)$ \\
\hline F11 & Mean(Std.) & $0.00 \mathrm{e}+000(0.00 \mathrm{e}+000)$ & $0.00 \mathrm{e}+000(0.00 \mathrm{e}+000)$ & $0.00 \mathrm{e}+000(0.00 \mathrm{e}+000)$ & $0.00 \mathrm{e}+000(0.00 \mathrm{e}+000)$ \\
\hline F12 & Mean(Std.) & $8.21 \mathrm{e}-003(1.74 \mathrm{e}-002)$ & $1.57 \mathrm{e}-032(5.59 \mathrm{e}-048)$ & $1.57 \mathrm{e}-032(5.59 \mathrm{e}-048)$ & $1.57 \mathrm{e}-032(5.59 \mathrm{e}-048)$ \\
\hline F13 & Mean(Std.) & $1.35 e-032(5.59 e-048)$ & $1.35 e-032(5.59 e-048)$ & $1.35 e-032(5.59 e-048)$ & $1.35 e-032(5.59 e-048)$ \\
\hline
\end{tabular}


The average time is also recorded. In this experiment VTR is chosen as 1e-8. Moreover, in the present work, the effect of the common controlling parameters of the algorithm i.e. population size and number of generations on the performance of the algorithm are also investigated by considering different population sizes and number of generations (function evaluation).

\section{Table 2}

Comparative statistical results of 13 benchmark function of E-BSADE for different values of population size (PS) at $\mathrm{D}=50$, over 25 runs, after 200000 function evaluations.

\begin{tabular}{|c|c|c|c|c|c|}
\hline Function & & $\mathrm{PS}=20$ & $\mathrm{PS}=50$ & $\mathrm{PS}=70$ & $\mathrm{PS}=100$ \\
\hline F1 & Mean(Std.) & $1.69 \mathrm{e}-050(8.43 \mathrm{e}-050)$ & $1.16 \mathrm{e}-096(2.80 \mathrm{e}-096)$ & $1.13 e-060(2.65 e-060)$ & $4.08 \mathrm{e}-037(1.42 \mathrm{e}-036)$ \\
\hline $\mathrm{F} 2$ & Mean(Std.) & $2.08 \mathrm{e}-006(1.04 \mathrm{e}-005)$ & $1.61 \mathrm{e}-048(3.31 \mathrm{e}-048)$ & $3.67 e-031(5.45 e-031)$ & $8.50 \mathrm{e}-020(1.06 \mathrm{e}-019)$ \\
\hline F3 & Mean(Std.) & $4.41 \mathrm{e}-006(2.21 \mathrm{e}-005)$ & $1.18 \mathrm{e}-080(5.69 \mathrm{e}-080)$ & $2.41 \mathrm{e}-051(7.35 \mathrm{e}-051)$ & $1.45 e-030(4.41 e-030)$ \\
\hline $\mathrm{F} 4$ & Mean(Std.) & $1.25 e-138(4.33 e-138)$ & $1.91 \mathrm{e}-039(8.92 \mathrm{e}-039)$ & $5.80 \mathrm{e}-023(2.55 \mathrm{e}-022)$ & $1.97 \mathrm{e}-014(4.45 \mathrm{e}-014)$ \\
\hline F5 & Mean(Std.) & $4.84 \mathrm{e}+001(1.09 \mathrm{e}-001)$ & $4.81 \mathrm{e}+001(1.96 \mathrm{e}-001)$ & $4.79 \mathrm{e}+001(2.05 \mathrm{e}-001)$ & $4.77 \mathrm{e}+001(3.37 \mathrm{e}-001)$ \\
\hline F6 & Mean(Std.) & $0.00 \mathrm{e}+000(0.00 \mathrm{e}+000)$ & $0.00 \mathrm{e}+000(0.00 \mathrm{e}+000)$ & $0.00 \mathrm{e}+000(0.00 \mathrm{e}+000)$ & $0.00 \mathrm{e}+000(0.00 \mathrm{e}+000)$ \\
\hline F7 & Mean(Std.) & $3.19 \mathrm{e}-004(1.57 \mathrm{e}-004)$ & $8.21 \mathrm{e}-004(3.19 \mathrm{e}-004)$ & $1.43 \mathrm{e}-003(6.16 \mathrm{e}-004)$ & $2.10 \mathrm{e}-003(7.72 \mathrm{e}-004)$ \\
\hline F8 & Mean(Std.) & $5.29 \mathrm{e}-001(3.95 \mathrm{e}-001)$ & $4.94 \mathrm{e}-001(4.95 \mathrm{e}-001)$ & $4.86 \mathrm{e}-001(4.32 \mathrm{e}-001)$ & $3.20 \mathrm{e}-001(2.93 \mathrm{e}-001)$ \\
\hline F9 & Mean(Std.) & $1.52 \mathrm{e}+001(3.27 \mathrm{e}+001)$ & $1.01 \mathrm{e}+000(4.35 \mathrm{e}+000)$ & $3.35 \mathrm{e}-001(9.28 \mathrm{e}-001)$ & $7.96 e-002(2.75 e-001)$ \\
\hline F10 & Mean(Std.) & $1.08 \mathrm{e}-001(5.41 \mathrm{e}-001)$ & $2.03 e-015(1.69 e-015)$ & $3.16 \mathrm{e}-015(1.74 \mathrm{e}-015)$ & $1.39 e-011(5.23 e-011)$ \\
\hline F11 & Mean(Std.) & $3.64 \mathrm{e}-003(1.82 \mathrm{e}-002)$ & $0.00 \mathrm{e}+000(0.00 \mathrm{e}+000)$ & $0.00 \mathrm{e}+000(0.00 \mathrm{e}+000)$ & $0.00 \mathrm{e}+000(0.00 \mathrm{e}+000)$ \\
\hline F12 & Mean(Std.) & $3.30 \mathrm{e}-002(5.69 \mathrm{e}-002)$ & $9.42 \mathrm{e}-033(1.40 \mathrm{e}-048)$ & $9.42 \mathrm{e}-033(1.40 \mathrm{e}-048)$ & $9.42 \mathrm{e}-033(1.40 \mathrm{e}-048)$ \\
\hline F13 & Mean(Std.) & $6.68 \mathrm{e}-029(3.34 \mathrm{e}-028)$ & $1.35 e-032(5.59 e-048)$ & $1.35 e-032(5.59 e-048)$ & $1.35 e-032(5.59 e-048)$ \\
\hline
\end{tabular}

Table 1 and 2 shows the effect of population size on the performance of the algorithm, the algorithm is experimented with different population sizes viz. 20, 50, 70 and 100 and dimension viz. 30 and 50 with function evaluations in each strategy is 200000 (for function F1 to F13). For the considered test problems, the E-DEBSA algorithm runs 25 times for each benchmark function. Boldface represents the best solution found after reaching the maximum number of function evaluations. From Table 1, it is observed that for functions F1, F4, and F7, strategy with population size of 20 and number of function evaluations 200000 produced the best results than the other strategies. For functions F2, F3, F9, and F10, strategy with population size of 50 gave the best results. For functions F8, strategy with population size of 70 and for functions F5, strategy with population size of 100 produced the best results. For functions F6, F11 and F13 strategies produced the same results and hence no effect of population size on these functions to achieve their respective global optimum values with same number of function evaluations. For function F12, strategy with population size 50,70 and 100 produced the identical results. Similarly, it is observed from Table 2 that for functions F4 and F7 strategy with population size 20 produced best results than the other strategies. For functions F1, F2, F3, and F10, strategy with population size 50 produced best results than the other strategies. For functions F5, F8, and F9, strategy with population size 100 produced best results than the other strategies. For functions F6, strategies produced the same results and hence no effect of population size on these functions to achieve their respective global optimum values with same number of function evaluations. For function F11, F12, and F13 strategy with population size 50, 70 and 100 produced the identical results.

\section{Table 3}

Comparative statistical results of 8 benchmark function (F14-F21) of E-BSADE for different values of population size (PS) over 30 runs, after 10000 function evaluation.

\begin{tabular}{|c|c|c|c|c|c|}
\hline Function & & $\mathrm{PS}=20$ & $\mathrm{PS}=50$ & $\mathrm{PS}=70$ & $\mathrm{PS}=100$ \\
\hline F14 & Mean(Std.) & $3.34 \mathrm{e}-002(1.81 \mathrm{e}-001)$ & $2.00 \mathrm{e}-010(7.36 \mathrm{e}-010)$ & $2.98 \mathrm{e}-011(5.90 \mathrm{e}-011)$ & $4.19 \mathrm{e}-011(8.93 \mathrm{e}-011)$ \\
\hline F15 & Mean(Std.) & $8.41 \mathrm{e}-004(8.40 \mathrm{e}-004)$ & $9.77 \mathrm{e}-005(1.22 \mathrm{e}-004)$ & $1.61 \mathrm{e}-004(1.19 \mathrm{e}-004)$ & $1.98 \mathrm{e}-004(1.16 \mathrm{e}-004)$ \\
\hline F16 & Mean(Std.) & $1.15 \mathrm{e}-004(5.43 \mathrm{e}-004)$ & $4.65 \mathrm{e}-008(1.12 \mathrm{e}-016)$ & $4.65 e-008(1.06 e-016)$ & $4.65 \mathrm{e}-008(2.52 \mathrm{e}-015)$ \\
\hline F17 & Mean(Std.) & $9.22 \mathrm{e}-005(4.65 \mathrm{e}-004)$ & $7.36 \mathrm{e}-006(0.00 \mathrm{e}+000)$ & $7.36 e-006(0.00 e+000)$ & 7.36e-006(1.10e-012) \\
\hline F18 & Mean(Std.) & $6.30 \mathrm{e}-002(3.45 \mathrm{e}-001)$ & $0.00 \mathrm{e}+000(0.00 \mathrm{e}+000)$ & $0.00 \mathrm{e}+000(0.00 \mathrm{e}+000)$ & $0.00 \mathrm{e}+000(0.00 \mathrm{e}+000)$ \\
\hline F19 & Mean(Std.) & $2.26 \mathrm{e}+000(3.11 \mathrm{e}+000)$ & $7.57 \mathrm{e}-001(2.00 \mathrm{e}+000)$ & $1.68 \mathrm{e}-001(9.22 \mathrm{e}-001)$ & $2.38 \mathrm{e}-001(9.26 \mathrm{e}-001)$ \\
\hline F21 & Mean(Std.) & $1.35 \mathrm{e}+000(2.78 \mathrm{e}+000)$ & $4.34 \mathrm{e}-001(1.68 \mathrm{e}+000)$ & $6.09 \mathrm{e}-003(3.34 \mathrm{e}-002)$ & $1.98 \mathrm{e}-002(5.71 \mathrm{e}-002)$ \\
\hline
\end{tabular}


Table 3 also shows the effect of population size on the performance of the algorithm, the algorithm is experimented with different population sizes viz. 20, 50, 70 and 100 with function evaluations in each strategy 10000 (for function F14 to F21). For the considered test problems, the E-DEBSA algorithm runs 30 times for each benchmark function. It is observed from Table 3 that for functions F16, strategy with population size of 50 produced the best results than the other strategies. For functions F14, F15, F19, F20, and F21 strategy with population size of 70 produced the best results than the other strategies. For function F17, and F18 strategy with population size 50, 70 and 100 produced the identical results.

\section{Table 4}

Comparative statistical results of 21 benchmark function of E-BSADE with two other state-of-the-art algorithms

\begin{tabular}{|c|c|c|c|c|}
\hline Function & & E-BSADE & BSA & $\mathrm{DE}$ \\
\hline F1 & Mean(Std.) & $1.89 \mathrm{e}-095(6.10 \mathrm{e}-095)$ & $1.40 \mathrm{e}-012(1.29 \mathrm{e}-012)$ & $4.98 \mathrm{e}-032(1.42 \mathrm{e}-031)$ \\
\hline $\mathrm{F} 2$ & Mean(Std.) & $6.15 e-048(2.24 e-047)$ & $7.45 e-008(4.77 e-008)$ & $1.77 \mathrm{e}-019(2.23 \mathrm{e}-019)$ \\
\hline F3 & Mean(Std.) & $4.70 e-081(1.53 e-080)$ & $1.24 \mathrm{e}-011(9.48 \mathrm{e}-012)$ & $3.28 \mathrm{e}-031(5.24 \mathrm{e}-031)$ \\
\hline F4 & Mean(Std.) & $3.29 \mathrm{e}-040(1.31 \mathrm{e}-039)$ & $5.67 \mathrm{e}+000(1.28 \mathrm{e}+000)$ & $2.20 \mathrm{e}+001(4.86 \mathrm{e}+000)$ \\
\hline F5 & Mean(Std.) & $4.80 \mathrm{e}+001(2.42 \mathrm{e}-001)$ & $9.10 \mathrm{e}+001(3.68 \mathrm{e}+001)$ & $1.13 \mathrm{e}+002(1.62 \mathrm{e}+002)$ \\
\hline F6 & Mean(Std.) & $0.00 \mathrm{e}+000(0.00 \mathrm{e}+000)$ & $0.00 \mathrm{e}+000(0.00 \mathrm{e}+000)$ & $2.68 \mathrm{e}+000(2.58 \mathrm{e}+000)$ \\
\hline F8 & Mean(Std.) & $3.44 \mathrm{e}-001(3.33 \mathrm{e}-001)$ & $6.24 \mathrm{e}+001(1.07 \mathrm{e}+002)$ & $2.54 \mathrm{e}-001(2.18 \mathrm{e}-001)$ \\
\hline F9 & Mean(Std.) & $3.18 \mathrm{e}-001(8.96 \mathrm{e}-001)$ & $1.03 \mathrm{e}+001(2.95 \mathrm{e}+000)$ & $3.44 \mathrm{e}+001(8.49 \mathrm{e}+000)$ \\
\hline F10 & Mean(Std.) & $2.45 e-015(1.80 e-015)$ & $5.21 \mathrm{e}-007(7.66 \mathrm{e}-007)$ & $1.22 \mathrm{e}-001(3.41 \mathrm{e}-001)$ \\
\hline F11 & Mean(Std.) & $0.00 \mathrm{e}+000(0.00 \mathrm{e}+000)$ & $4.84 \mathrm{e}-012(1.90 \mathrm{e}-011)$ & $4.04 \mathrm{e}-003(7.47 \mathrm{e}-003)$ \\
\hline F12 & Mean(Std.) & $9.42 \mathrm{e}-033(1.40 \mathrm{e}-048)$ & $4.24 \mathrm{e}-013(9.02 \mathrm{e}-013)$ & $1.98 \mathrm{e}-002(5.68 \mathrm{e}-002)$ \\
\hline F13 & Mean(Std.) & $1.35 \mathrm{e}-032(5.59 \mathrm{e}-048)$ & $9.59 \mathrm{e}-014(1.28 \mathrm{e}-013)$ & $2.64 \mathrm{e}-003(9.13 \mathrm{e}-003)$ \\
\hline F14 & Mean(Std.) & $2.23 e-010(6.72 e-010)$ & $5.47 \mathrm{e}-009(1.20 \mathrm{e}-008)$ & $1.63 e-014(7.63 e-014)$ \\
\hline F16 & Mean(Std.) & $4.65 e-008(1.12 e-016)$ & $5.01 \mathrm{e}-008(6.28 \mathrm{e}-009)$ & $4.65 e-008(9.06 e-017)$ \\
\hline F17 & Mean(Std.) & $7.36 e-006(0.00 e+000)$ & $7.50 \mathrm{e}-006(2.64 \mathrm{e}-007)$ & $7.36 \mathrm{e}-006(0.00 \mathrm{e}+000)$ \\
\hline F18 & Mean(Std.) & $0.00 \mathrm{e}+000(0.00 \mathrm{e}+000)$ & $2.61 \mathrm{e}-007(5.50 \mathrm{e}-007)$ & $0.00 \mathrm{e}+000(0.00 \mathrm{e}+000)$ \\
\hline F19 & Mean(Std.) & $3.21 \mathrm{e}-007(3.51 \mathrm{e}-010)$ & $1.75 \mathrm{e}-001(3.08 \mathrm{e}-001)$ & $3.21 \mathrm{e}-007(7.25 \mathrm{e}-016)$ \\
\hline F20 & Mean(Std.) & $2.69 \mathrm{e}-001(1.34 \mathrm{e}+000)$ & $1.17 \mathrm{e}-001(1.45 \mathrm{e}-001)$ & $6.77 e-009(7.17 e-009)$ \\
\hline F21 & Mean(Std.) & $2.14 \mathrm{e}-001(1.07 \mathrm{e}+000)$ & $2.16 \mathrm{e}-001(3.78 \mathrm{e}-001)$ & $2.22 \mathrm{e}-009(2.11 \mathrm{e}-009)$ \\
\hline
\end{tabular}

The comparative results of each benchmark function given in Appendix 1 are presented in Tables 4-7 in the form of best solution. For each algorithm and each test function, 25 independent runs were conducted with 200000 function evaluations (FES) for function (F1 to F13) and 10000 function evaluations (FES) for function (F14 to F21) as the termination criterion. The population size was set to 50. Table 4 shows the comparison result of the proposed algorithm with the basic DE and BSA; boldface represents the best solution found after reaching the maximum number of function evaluations. From this table it is seen that the proposed algorithm found better performance in most of the cases from the other two algorithms. Also it is observed that that the proposed algorithm found better performance except for function no F8, F14, F20 and F21. The successful runs (SR), successful performance (SP) and average time are recorded which are given in Table 5, compared to basic DE, BSA.

Table 6 shows the comparison of the statistical result of the proposed algorithm with other DE mutation strategies; boldface represents the best solution found after reaching the maximum number of function evaluations. From this table it is seen that the proposed algorithm found better performance except for function no F5, F8, F14, F20, and F21. The successful runs (SR), successful performance (SP) and average time are recorded which are given in Table 7 compared others DE mutation strategies. Table 5 elicits that the proposed E-DEBSA has more superior perform than basic DE and BSA, as the SR values produced are generally higher than its peers in twelve different functions. Also in such case, the SP value as infinity, namely "Inf'. In Table 7 the proposed algorithm E-DEBSA also performs higher than others DE mutation strategies. 
Table 5

Comparative SR, SP and Average time of 21 benchmark function with BSA and DE algorithms.

\begin{tabular}{|c|c|c|c|c|c|c|c|c|c|}
\hline \multicolumn{2}{|c|}{ Function } & \multirow{2}{*}{$\begin{array}{l}\text { E-BSADE } \\
100\end{array}$} & \multirow{2}{*}{$\begin{array}{l}\text { BSA } \\
100\end{array}$} & \multirow{2}{*}{$\begin{array}{l}\mathrm{DE} \\
100\end{array}$} & \multicolumn{2}{|c|}{ Function } & \multirow{2}{*}{$\begin{array}{l}\text { E-BSADE } \\
96\end{array}$} & \multirow{2}{*}{$\begin{array}{l}\text { BSA } \\
100\end{array}$} & \multirow{2}{*}{$\begin{array}{l}\mathrm{DE} \\
76\end{array}$} \\
\hline F1 & SR & & & & F12 & SR & & & \\
\hline & SP & $2.82 \mathrm{E}+04$ & $1.49 \mathrm{E}+05$ & $6.93 \mathrm{E}+04$ & & SP & $3.36 \mathrm{E}+04$ & $1.40 \mathrm{E}+05$ & $1.73 \mathrm{E}+05$ \\
\hline & Avg.time & 1.33 & 13.65 & 2.54 & & Avg.time & 7.29 & 15.09 & 9.97 \\
\hline \multirow[t]{3}{*}{$\mathrm{F} 2$} & SR & 100 & 0 & 100 & \multirow[t]{3}{*}{ F13 } & SR & 100 & 100 & 88 \\
\hline & SP & $4.03 \mathrm{E}+04$ & Inf & $9.73 \mathrm{E}+04$ & & SP & $2.58 \mathrm{E}+04$ & $1.33 \mathrm{E}+05$ & $1.06 \mathrm{E}+05$ \\
\hline & Avg.time & 2.14 & 21.79 & 3.66 & & Avg.time & 5.06 & 13.54 & 6.27 \\
\hline \multirow[t]{3}{*}{ F3 } & SR & 100 & 100 & 100 & \multirow[t]{3}{*}{ F14 } & SR & 100 & 96 & 100 \\
\hline & SP & $3.51 \mathrm{E}+04$ & $1.65 \mathrm{E}+05$ & $7.60 \mathrm{E}+04$ & & SP & $1.84 \mathrm{E}+03$ & $7.33 \mathrm{E}+03$ & $2.87 \mathrm{E}+03$ \\
\hline & Avg.time & 3.02 & 19.27 & 3.99 & & Avg.time & 1.33 & 4.6 & 0.65 \\
\hline \multirow[t]{3}{*}{$\mathrm{F} 4$} & SR & 100 & 0 & 0 & \multirow[t]{3}{*}{ F15 } & SR & 4 & 0 & 8 \\
\hline & SP & $5.78 \mathrm{E}+04$ & Inf & Inf & & SP & $2.49 \mathrm{E}+05$ & Inf & $1.24 \mathrm{E}+05$ \\
\hline & Avg.time & 3.27 & 16.49 & 7.55 & & Avg.time & 1.25 & 1.02 & 0.45 \\
\hline \multirow[t]{3}{*}{ F5 } & SR & 0 & 0 & 0 & \multirow[t]{3}{*}{ F16 } & SR & 0 & 0 & 0 \\
\hline & SP & Inf & Inf & Inf & & SP & Inf & Inf & Inf \\
\hline & Avg.time & 16.37 & 16.16 & 7.42 & & Avg.time & 0.88 & 0.71 & 0.36 \\
\hline \multirow[t]{3}{*}{ F6 } & SR & 100 & 100 & 40 & \multirow[t]{3}{*}{ F17 } & SR & 0 & 0 & 0 \\
\hline & SP & $1.27 \mathrm{E}+04$ & $5.25 \mathrm{E}+04$ & $3.34 \mathrm{E}+05$ & & SP & Inf & Inf & Inf \\
\hline & Avg.time & 1.23 & 4.43 & 5.1 & & Avg.time & 0.84 & 0.65 & 0.35 \\
\hline \multirow[t]{3}{*}{ F7 } & SR & 0 & 0 & 0 & \multirow[t]{3}{*}{ F18 } & SR & 100 & 60 & 100 \\
\hline & SP & Inf & Inf & Inf & & SP & $2.72 \mathrm{E}+03$ & $1.56 \mathrm{E}+04$ & $2.38 \mathrm{E}+03$ \\
\hline & Avg.time & 24.82 & 27.71 & 8.97 & & Avg.time & 0.23 & 0.64 & 0.09 \\
\hline \multirow[t]{3}{*}{ F8 } & SR & 0 & 0 & 0 & \multirow[t]{3}{*}{ F19 } & SR & 0 & 0 & 0 \\
\hline & SP & Inf & Inf & Inf & & SP & Inf & Inf & Inf \\
\hline & Avg.time & 21.79 & 20.35 & 8.53 & & Avg.time & 1.23 & 1.05 & 0.48 \\
\hline \multirow[t]{3}{*}{ F9 } & SR & 84 & 0 & 0 & \multirow[t]{3}{*}{ F20 } & SR & 84 & 0 & 84 \\
\hline & SP & $7.91 \mathrm{E}+04$ & Inf & Inf & & SP & $9.28 \mathrm{E}+03$ & Inf & $8.64 \mathrm{E}+03$ \\
\hline & Avg.time & 6.73 & 225.06 & 8.28 & & Avg.time & 0.93 & 1.08 & 0.35 \\
\hline \multirow[t]{3}{*}{ F10 } & SR & 100 & 0 & 92 & \multirow[t]{3}{*}{ F21 } & SR & 92 & 0 & 100 \\
\hline & SP & $7.97 \mathrm{E}+04$ & Inf & $1.24 \mathrm{E}+05$ & & SP & $7.50 \mathrm{E}+03$ & Inf & $5.52 \mathrm{E}+03$ \\
\hline & Avg.time & 8.74 & 16.15 & 4.68 & & Avg.time & 0.83 & 1.07 & 0.27 \\
\hline \multirow[t]{3}{*}{ F11 } & SR & 100 & 100 & 80 & & & & & \\
\hline & SP & $3.03 \mathrm{E}+04$ & $1.53 \mathrm{E}+05$ & $1.20 \mathrm{E}+05$ & & & & & \\
\hline & Avg.time & 3.25 & 7.02 & 3.85 & & & & & \\
\hline
\end{tabular}

\section{Table 6}

Comparative statistical results of 21 benchmark function of E-BSADE with respect to other DE Mutation Strategies.

\begin{tabular}{|c|c|c|c|c|c|}
\hline Function & & $\mathrm{DE} /$ target to best/1/bin & $\mathrm{DE} /$ Current to $\mathrm{rand} / 1 /$ bin & DE/best/2/bin & E-BSADE \\
\hline F1 & Mean(Std.) & $6.60 \mathrm{e}+003(1.88 \mathrm{e}+003)$ & $2.27 \mathrm{e}+000(4.12 \mathrm{e}+000)$ & $1.93 \mathrm{e}-048(5.49 \mathrm{e}-048)$ & $1.89 \mathrm{e}-095(6.10 \mathrm{e}-095)$ \\
\hline $\mathrm{F} 2$ & Mean(Std.) & $4.51 \mathrm{e}+001(8.46 \mathrm{e}+000)$ & $1.05 \mathrm{e}-002(2.90 \mathrm{e}-002)$ & $2.03 e-024(8.77 e-024)$ & $6.15 e-048(2.24 e-047)$ \\
\hline F3 & Mean(Std.) & $1.52 \mathrm{e}+005(4.59 \mathrm{e}+004)$ & $4.78 \mathrm{e}+001(9.20 \mathrm{e}+001)$ & $1.09 e-046(3.77 e-046)$ & $4.70 e-081(1.53 e-080)$ \\
\hline F4 & Mean(Std.) & $2.16 \mathrm{e}+001(3.07 \mathrm{e}+000)$ & $1.31 \mathrm{e}+001(3.55 \mathrm{e}+000)$ & $1.95 \mathrm{e}+001(3.44 \mathrm{e}+000)$ & $3.29 \mathrm{e}-040(1.31 \mathrm{e}-039)$ \\
\hline F5 & Mean(Std.) & $2.06 \mathrm{e}+006(8.99 \mathrm{e}+005)$ & $3.24 \mathrm{e}+002(4.30 \mathrm{e}+002)$ & $4.12 \mathrm{e}+001(3.79 \mathrm{e}+001)$ & $4.80 \mathrm{e}+001(2.42 \mathrm{e}-001)$ \\
\hline F6 & Mean(Std.) & $5.93 \mathrm{e}+003(1.01 \mathrm{e}+003)$ & $1.92 \mathrm{e}+001(2.21 \mathrm{e}+001)$ & $3.87 \mathrm{e}+001(2.72 \mathrm{e}+001)$ & $0.00 \mathrm{e}+000(0.00 \mathrm{e}+000)$ \\
\hline F7 & Mean(Std.) & $3.95 \mathrm{e}-001(2.59 \mathrm{e}-001)$ & $1.24 \mathrm{e}-002(6.70 \mathrm{e}-003)$ & $2.92 \mathrm{e}-002(1.22 \mathrm{e}-002)$ & $1.05 e-003(3.79 e-004)$ \\
\hline F8 & Mean(Std.) & $4.28 \mathrm{e}+001(2.14 \mathrm{e}+002)$ & $2.95 e-001(3.17 e-001)$ & $2.74 \mathrm{e}-001(2.61 \mathrm{e}-001)$ & $3.44 \mathrm{e}-001(3.33 \mathrm{e}-001)$ \\
\hline F9 & Mean(Std.) & $1.38 \mathrm{e}+002(2.69 \mathrm{e}+001)$ & $3.22 \mathrm{e}+002(1.53 \mathrm{e}+001)$ & $1.96 \mathrm{e}+002(1.39 \mathrm{e}+002)$ & $3.18 e-001(8.96 e-001)$ \\
\hline F10 & Mean(Std.) & $1.16 \mathrm{e}+001(9.51 \mathrm{e}-001)$ & $1.26 \mathrm{e}+000(6.09 \mathrm{e}-001)$ & $2.82 \mathrm{e}+000(8.00 \mathrm{e}-001)$ & $2.45 \mathrm{e}-015(1.80 \mathrm{e}-015)$ \\
\hline F11 & Mean(Std.) & $6.33 \mathrm{e}+001(1.33 \mathrm{e}+001)$ & $9.35 \mathrm{e}-002(1.13 \mathrm{e}-001)$ & $1.75 \mathrm{e}-002(2.05 \mathrm{e}-002)$ & $0.00 \mathrm{e}+000(0.00 \mathrm{e}+000)$ \\
\hline F12 & Mean(Std.) & $2.40 \mathrm{e}+004(9.50 \mathrm{e}+004)$ & $1.81 \mathrm{e}-001(2.26 \mathrm{e}-001)$ & $6.49 \mathrm{e}-001(1.12 \mathrm{e}+000)$ & $9.42 \mathrm{e}-033(1.40 \mathrm{e}-048)$ \\
\hline F13 & Mean(Std.) & $7.65 e+005(1.49 e+006)$ & $5.64 \mathrm{e}-002(1.20 \mathrm{e}-001)$ & $4.32 \mathrm{e}+001(5.67 \mathrm{e}+001)$ & $1.35 e-032(5.59 e-048)$ \\
\hline F14 & Mean(Std.) & $4.08 \mathrm{e}-014(1.15 \mathrm{e}-013)$ & $1.28 \mathrm{e}-007(4.43 \mathrm{e}-007)$ & $2.47 e-014(7.10 e-014)$ & $2.23 \mathrm{e}-010(6.72 \mathrm{e}-010)$ \\
\hline F16 & Mean(Std.) & $4.65 \mathrm{e}-008(1.09 \mathrm{e}-016)$ & $4.65 e-008(5.81 e-014)$ & $4.65 \mathrm{e}-008(1.02 \mathrm{e}-016)$ & $4.65 \mathrm{e}-008(1.12 \mathrm{e}-016)$ \\
\hline F17 & Mean(Std.) & $7.36 \mathrm{e}-006(0.00 \mathrm{e}+000)$ & $7.51 \mathrm{e}-006(4.76 \mathrm{e}-007)$ & $7.36 \mathrm{e}-006(0.00 \mathrm{e}+000)$ & $7.36 \mathrm{e}-006(0.00 \mathrm{e}+000)$ \\
\hline F18 & Mean(Std.) & $0.00 \mathrm{e}+000(0.00 \mathrm{e}+000)$ & $0.00 \mathrm{e}+000(0.00 \mathrm{e}+000)$ & $0.00 \mathrm{e}+000(0.00 \mathrm{e}+000)$ & $0.00 \mathrm{e}+000(0.00 \mathrm{e}+000)$ \\
\hline F19 & Mean(Std.) & $2.70 \mathrm{e}+000(3.68 \mathrm{e}+000)$ & $2.08 \mathrm{e}-004(6.75 \mathrm{e}-004)$ & $3.43 \mathrm{e}+000(2.98 \mathrm{e}+000)$ & $3.21 \mathrm{e}-007(3.51 \mathrm{e}-010)$ \\
\hline F20 & Mean(Std.) & $2.01 \mathrm{e}+000(3.32 \mathrm{e}+000)$ & $1.10 \mathrm{e}-008(9.04 \mathrm{e}-009)$ & $5.16 \mathrm{e}-001(1.82 \mathrm{e}+000)$ & $2.69 \mathrm{e}-001(1.34 \mathrm{e}+000)$ \\
\hline $\mathrm{F} 21$ & Mean(Std.) & $8.99 \mathrm{e}-001(2.49 \mathrm{e}+000)$ & $2.79 \mathrm{e}-006(1.40 \mathrm{e}-005)$ & $2.19 \mathrm{e}+000(3.34 \mathrm{e}+000)$ & $2.14 \mathrm{e}-001(1.07 \mathrm{e}+000)$ \\
\hline
\end{tabular}


Table 7

Comparative SR. SP and Average time of 21 benchmark function with other DE mutation strategies.

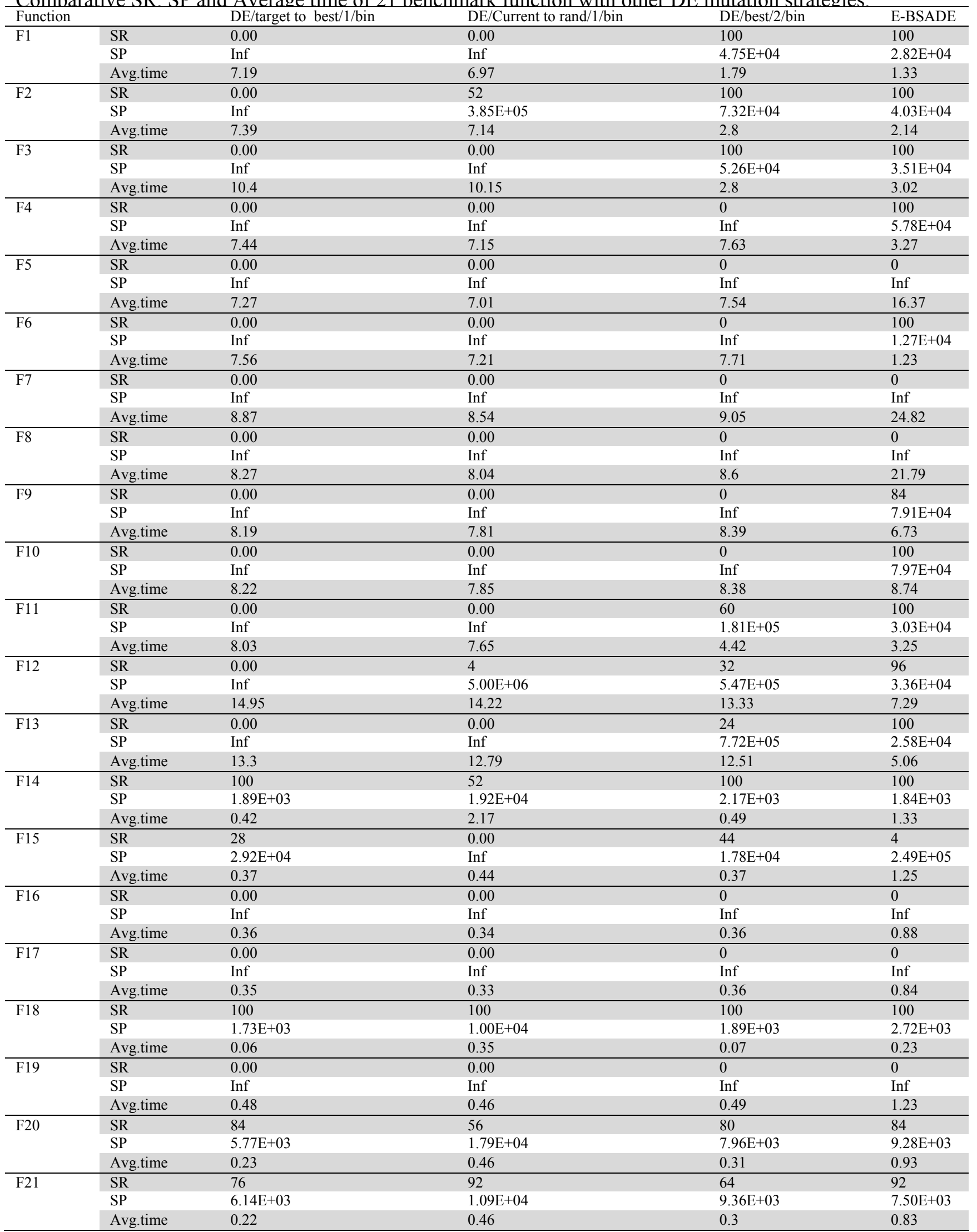


Table 8

Comparative statistical results of 13 benchmark function of E-BSADE with other state-of-the-art PSO variants.

\begin{tabular}{|c|c|c|c|c|c|c|}
\hline Function & Mean(Std.) & FDR-PSO & CPSO-H & UPSO & CLPSO & E-BSADE \\
\hline F1 & Mean(Std.) & $4.90 \mathrm{e}-045(2.03 \mathrm{e}-044)$ & $1.63 \mathrm{e}-004(1.14 \mathrm{e}-004)$ & $9.64 \mathrm{e}-039(8.30 \mathrm{e}-039)$ & $8.82 \mathrm{e}-007(3.26 \mathrm{e}-007)$ & $6.71 e-076(2.17 e-075)$ \\
\hline F2 & Mean(Std.) & $1.86 \mathrm{e}-015(8.24 \mathrm{e}-015)$ & $3.28 \mathrm{e}-003(7.49 \mathrm{e}-004)$ & $3.51 \mathrm{e}-023(2.31 \mathrm{e}-023)$ & $9.69 \mathrm{e}-005(1.54 \mathrm{e}-005)$ & $1.74 \mathrm{e}-038(3.79 \mathrm{e}-038)$ \\
\hline F3 & Mean(Std.) & $3.92 \mathrm{e}-043(1.58 \mathrm{e}-042)$ & $2.51 \mathrm{e}-003(1.45 \mathrm{e}-003)$ & $2.99 \mathrm{e}-037(3.53 \mathrm{e}-037)$ & $1.81 \mathrm{e}-005(7.36 \mathrm{e}-006)$ & $2.50 e-065(8.55 e-065)$ \\
\hline F4 & Mean(Std.) & $6.22 \mathrm{e}-001(2.29 \mathrm{e}-001)$ & $1.06 \mathrm{e}+001(3.55 \mathrm{e}+000)$ & $3.02 \mathrm{e}-001(1.96 \mathrm{e}-001)$ & $1.62 \mathrm{e}+001(1.21 \mathrm{e}+000)$ & $1.40 \mathrm{e}-030(4.88 \mathrm{e}-030)$ \\
\hline F5 & Mean(Std.) & $3.69 \mathrm{e}+001(3.03 \mathrm{e}+001)$ & $3.99 \mathrm{e}+001(3.47 \mathrm{e}+001)$ & $4.54 \mathrm{e}+001(2.70 \mathrm{e}+001)$ & $8.84 e+001(3.66 e+001)$ & $3.81 \mathrm{e}+001(1.45 \mathrm{e}-001)$ \\
\hline F6 & Mean(Std.) & $1.65 \mathrm{e}+000(1.39 \mathrm{e}+000)$ & $0.00 \mathrm{e}+000(0.00 \mathrm{e}+000)$ & $0.00 \mathrm{e}+000(0.00 \mathrm{e}+000)$ & $0.00 e+000(0.00 e+000)$ & $0.00 \mathrm{e}+000(0.00 \mathrm{e}+000)$ \\
\hline F7 & Mean(Std.) & $6.99 \mathrm{e}-003(2.48 \mathrm{e}-003)$ & $3.31 \mathrm{e}-002(1.51 \mathrm{e}-002)$ & $2.42 \mathrm{e}-002(7.32 \mathrm{e}-003)$ & $1.81 \mathrm{e}-002(4.60 \mathrm{e}-003)$ & $1.10 e-003(3.73 e-004)$ \\
\hline F8 & Mean(Std.) & $3.67 \mathrm{e}+003(1.15 \mathrm{e}+003)$ & $4.15 e+001(6.95 e+001)$ & $2.62 \mathrm{e}-004(5.60 \mathrm{e}-004)$ & $1.79 \mathrm{e}-002(1.26 \mathrm{e}-002)$ & $5.45 \mathrm{e}-001(3.55 \mathrm{e}-001)$ \\
\hline F9 & Mean(Std.) & $5.26 \mathrm{e}+001(8.87 \mathrm{e}+000)$ & $4.18 \mathrm{e}-005(2.64 \mathrm{e}-005)$ & $9.02 \mathrm{e}+001(1.87 \mathrm{e}+001)$ & $1.96 \mathrm{e}-001(1.30 \mathrm{e}-001)$ & $1.52 \mathrm{e}-001(4.95 \mathrm{e}-001)$ \\
\hline F11 & Mean(Std.) & $9.22 \mathrm{e}-003(1.34 \mathrm{e}-002)$ & $1.30 \mathrm{e}-002(2.40 \mathrm{e}-002)$ & $4.44 \mathrm{e}-017(1.99 \mathrm{e}-016)$ & $1.66 \mathrm{e}-005(8.02 \mathrm{e}-006)$ & $0.00 \mathrm{e}+000(0.00 \mathrm{e}+000)$ \\
\hline F12 & Mean(Std.) & $3.49 \mathrm{e}-002(6.03 \mathrm{e}-002)$ & $1.43 \mathrm{e}-006(1.32 \mathrm{e}-006)$ & $4.80 \mathrm{e}-003(2.15 \mathrm{e}-002)$ & $7.86 e-004(4.17 e-004)$ & $1.18 \mathrm{e}-032(1.40 \mathrm{e}-048)$ \\
\hline F13 & Mean(Std.) & $2.75 e-003(4.88 e-003)$ & $3.91 e-006(4.80 e-006)$ & $1.35 \mathrm{e}-032(2.81 \mathrm{e}-048)$ & $5.91 \mathrm{e}-007(1.04 \mathrm{e}-007)$ & $1.35 \mathrm{e}-032(2.81 \mathrm{e}-048)$ \\
\hline
\end{tabular}

The performance of E-DEBSA is also compared with the different PSO variants like FDR-PSO (Peram et al., 2003), CPSO-H (Van den Bergh \& Engelbrecht, 2004), UPSO (Parsopoulos \& Vrahatis, 2004), and CLPSO (Liang et al., 2006) for the same number of function evaluations. The comparative results of each 13 benchmark function (F1 to F13) are presented in Tables 8 in the form of average solution and standard deviation obtained in 20 independent runs on each benchmark function with the population size is 50 , dimension (D) is 40 and maximum number of function evaluation is 150000 . The boldface value given in parenthesis indicates the global optimum value of that function. From Table 8 it is observed that the proposed method perform best solution except for function F5, F8 and F9. Also for F6 and F11, EDEBSA reaches the global optimum solution. Fig. 1(a)-(1) shows the convergence graph of E-DEBSA. To be specific, we observe that the convergence curve of E-DEBSA in functions F1-F4 (Fig. 1(a)-(d)) linearly increased to the optimum point. For function F5, Fig.1.(e) the converges graph curvilinear increased to optimum point. Also for F6-F13, the converges graph Fig.1(f)-Fig.1.(1), the proposed method E-DEBSA converges faster to the global optimum.

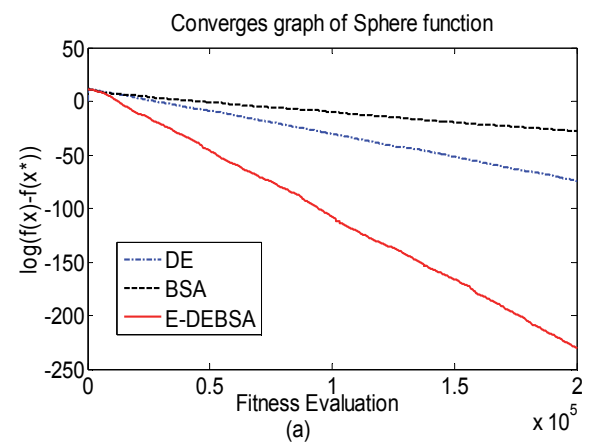

F1

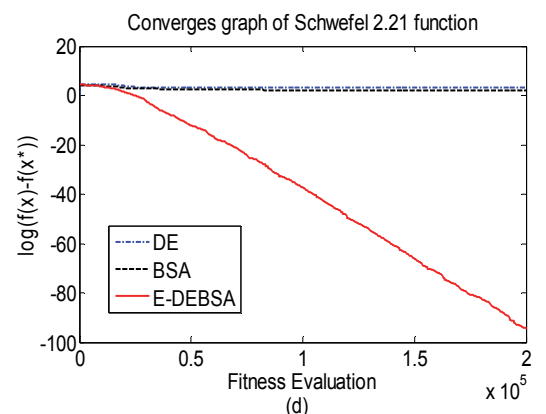

F4

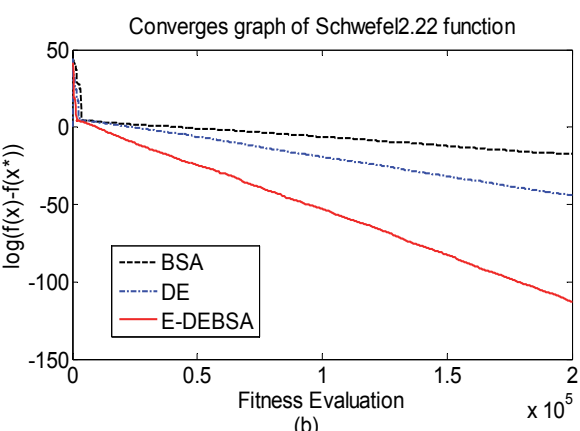

F2

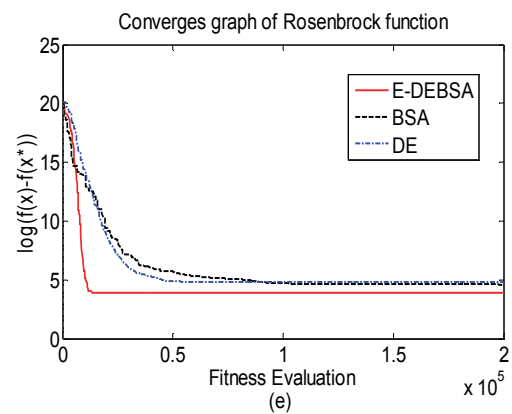

F5

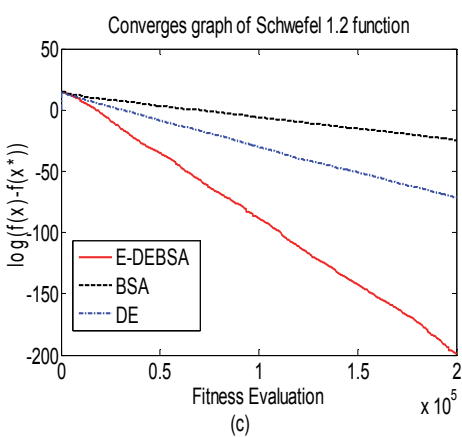

F3

Converges graph of Step function

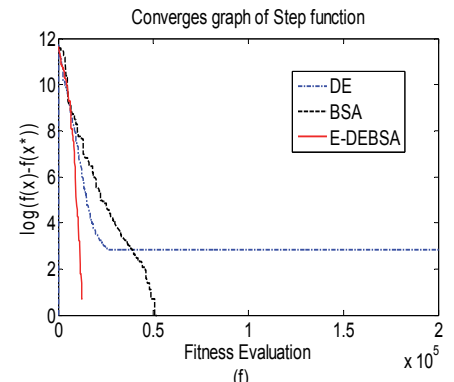

F6 


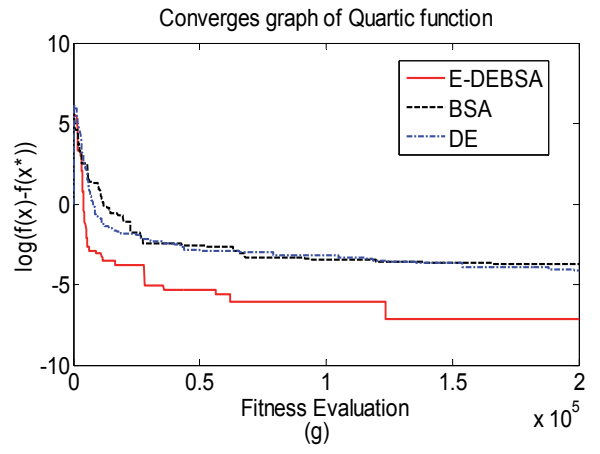

F7

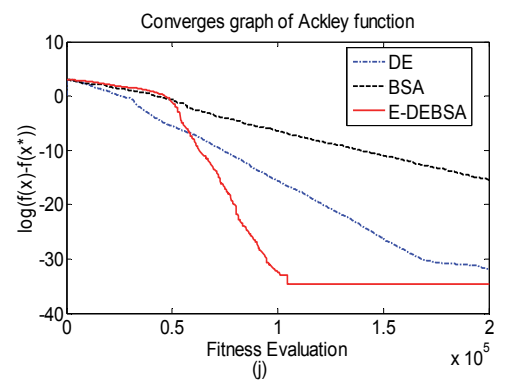

F10

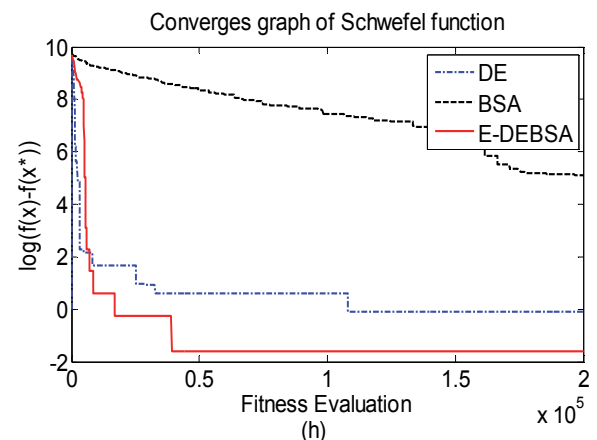

F8

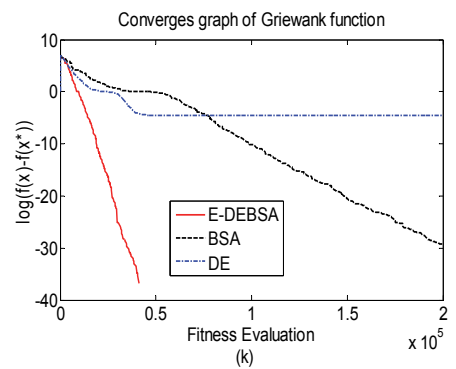

F11

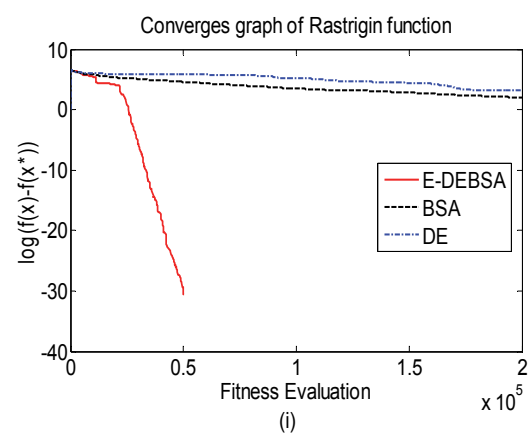

F9

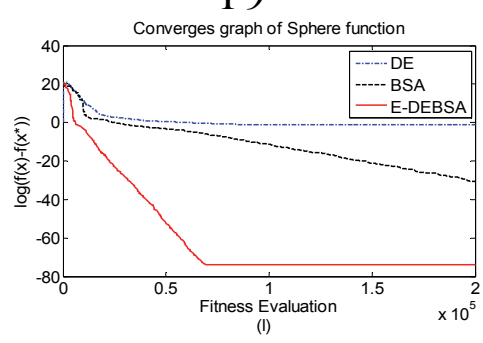

F12

Fig. 1. Convergence curves of 50 dimensional test functions: (a) F1, (b) F2, (c) F3, (d) F4, (e) F5, (f) F6, (g) F7, (h) F8, (i) F9, (j) F10, (k) F11, and (1) F12 .

\section{Conclusions}

Since the combination of two algorithms can provide a higher efficiency and flexibility when dealing with real-world and large scale problems, this paper proposed an ensemble algorithm of DE and BSA called E-DEBSA. For the validity of the proposed method, 21 different benchmark functions have been considered and the result has been compared to basic DE, BSA and different DE mutation strategies. Also the result are compared with different PSO variants. Moreover, the effect of common controlling parameters (i.e. population size, and number of generations) on the performance of E-DEBSA algorithm has also been investigated by considering different combinations of common controlling parameters. Also, in general, the strategy with higher population size has produced better results than that with smaller population size for same number of function evaluations for lower dimension and strategy with lower population size has produced better results than that with higher population size for same number of function evaluations for higher dimension. From the above experimental results and discussion it can be suggeste that its overall performance was better than the other competitors.

\section{References}

Behnamian, J., Zandieh, M., \& Ghomi, S. F. (2009). Parallel-machine scheduling problems with sequence-dependent setup times using an ACO, SA and VNS hybrid algorithm. Expert Systems with Applications, 36(6), 9637-9644.

Blum, C. (2005). Ant colony optimization: Introduction and recent trends.Physics of Life reviews, 2(4), 353-373.

Civicioglu, P. (2013). Backtracking search optimization algorithm for numerical optimization problems. Applied Mathematics and Computation, 219(15), 8121-8144.

Das, S. K. (2005). Slope stability analysis using genetic algorithm. The Electronic Journal of Geotechnical Engineering, 10, 429-439. 
Eskandar, H., Sadollah, A., Bahreininejad, A., \& Hamdi, M. (2012). Water cycle algorithm-A novel metaheuristic optimization method for solving constrained engineering optimization problems. Computers \& Structures, 110, 151-166.

Fan, S. K. S., \& Zahara, E. (2007). A hybrid simplex search and particle swarm optimization for unconstrained optimization. European Journal of Operational Research, 181(2), 527-548.

Gämperle, R., Müller, S. D., \& Koumoutsakos, P. (2002). A parameter study for differential evolution. Advances in Intelligent Systems, Fuzzy Systems, Evolutionary Computation, 10, 293-298.

Glover, F., \& Laguna, M. (1997). Tabu Search, Kluwer Academic Publishers, Norwell, MA.

Gong, W., Cai, Z., \& Ling, C.X. (2010). DE/BBO: a hybrid differential evolution with biogeographybased optimization for global numerical optimization. Soft Computing, 15(4), 645-665.

Guo, W., Li, W., Zhang, Q., Wang, L., Wu, Q., \& Ren, H. (2014). Biogeography-based particle swarm optimization with fuzzy elitism and its applications to constrained engineering problems. Engineering Optimization, 46(11), 1465-1484.

Zhang, J. R., Zhang, J., Lok, T. M., \& Lyu, M. R. (2007). A hybrid particle swarm optimization-backpropagation algorithm for feedforward neural network training. Applied Mathematics and Computation, 185(2), 1026-1037.

Kennedy, J., \& Eberhart, R.C. (1995). Particle swarm optimization, Proceedings of the 1995 IEEE International Conference on Neural Networks (Perth, Australia, IEEE Service Center, Piscataway, NJ, 1995), vol. 4, pp. 1942-1948.

Lampinen, J., \& Zelinka, I. (2000). On stagnation of the differential evolution algorithm, in: Proceedings of MENDEL 2000, 6th International Mendel Conference on Soft Computing, pp. 76-83.

Lee, K. S., \& Geem, Z. W. (2005). A new meta-heuristic algorithm for continuous engineering optimization: harmony search theory and practice.Computer Methods in Applied Mechanics and Engineering, 194(36), 3902-3933.

Liang, J. J., Qin, A. K., Suganthan, P. N., \& Baskar, S. (2006). Comprehensive learning particle swarm optimizer for global optimization of multimodal functions. Evolutionary Computation, IEEE Transactions on, 10(3), 281-295.

Lin, W. Y. (2010). A GA-DE hybrid evolutionary algorithm for path synthesis of four-bar linkage. Mechanism and Machine Theory, 45(8), 1096-1107.

Liu, H., Cai, Z., \& Wang, Y. (2010). Hybridizing particle swarm optimization with differential evolution for constrained numerical and engineering optimization.Applied Soft Computing, 10(2), 629-640.

Kao, Y. T., \& Zahara, E. (2008). A hybrid genetic algorithm and particle swarm optimization for multimodal functions. Applied Soft Computing, 8(2), 849-857.

Mallipeddi, R., Suganthan, P. N., Pan, Q. K., \& Tasgetiren, M. F. (2011). Differential evolution algorithm with ensemble of parameters and mutation strategies. Applied Soft Computing, 11(2), 1679-1696.

Nama, S., Saha, A. K., \& Ghosh, S. (2015). Parameters Optimization of Geotechnical Problem Using Different Optimization Algorithm, vol-33, Geotechnical and Geological Engineering, DOI 10.1007/s10706-015-9898-0

Nourelfath, M., Nahas, N., \& Montreuil, B. (2007). Coupling ant colony optimization and the extended great deluge algorithm for the discrete facility layout problem. Engineering Optimization, 39(8), 953968.

Parouha, R. P., Das, K. N., (2015), An efficient hybrid technique for numerical optimization and applications, Computers \& Industrial Engineering, 83, 193-216.

Parsopoulos, K. E., \& Vrahatis, M. N. (2004). UPSO: A unified particle swarm optimization scheme. Lecture Series on Computer and Computational Sciences, 1, 868-873.

Peram, T., Veeramachaneni, K., \& Mohan, C. K. (2003, April). Fitness-distance-ratio based particle swarm optimization. In Swarm Intelligence Symposium, 2003. SIS'03. Proceedings of the 2003 IEEE (pp. 174-181). IEEE.

Rahnamayan, S., Tizhoosh, H. R., \& Salama, M. (2008). Opposition-based differential evolution. Evolutionary Computation, IEEE Transactions on, 12(1), 64-79. 
Rao, R.V., \& Savsani, V.J. (2012). Mechanical Design Optimization using Advanced Optimization Technique, Springer Series in Advanced Manufacturing, Springer, London, Heidelberg.

Ronkkonen, J., Kukkonen, S., \& Price, K. V. (2005, September). Real-parameter optimization with differential evolution. In Proc. IEEE CEC (Vol. 1, pp. 506-513).

Sadollah, A., Bahreininejad, A., Eskandar, H., \& Hamdi, M. (2013). Mine blast algorithm: A new population based algorithm for solving constrained engineering optimization problems. Applied Soft Computing, 13(5), 2592-2612.

Sengupta, A., \& Upadhyay, A. (2009). Locating the critical failure surface in a slope stability analysis by genetic algorithm. Applied Soft Computing, 9(1), 387-392.

Shojaeefard, M. H., Khalkhali, A., Akbari, M., \& Tahani, M. (2013). Application of Taguchi optimization technique in determining aluminum to brass friction stir welding parameters. Materials \& Design, 52, 587-592.

Šmuc, T. (2002). Improving convergence properties of the differential evolution algorithm. Matoušek and Ošmera [529], 80-86.

Storn, R.(1996). On the usage of differential evolution for function optimization, in: Biennial Conference of the North American Fuzzy Information Processing Society (NAFIPS), IEEE, Berkeley, pp. 519523.

Storn, R., \& Price, K. (1995). Differential evolution-a simple and efficient adaptive scheme for global optimization over continuous spaces (Vol. 3). Berkeley: ICSI.

Storn, R., \& Price, K. (1997). Differential evolution-a simple and efficient heuristic for global optimization over continuous spaces. Journal of Global Optimization, 11(4), 341-359.

Suganthan, P. N., Hansen, N., Liang, J. J., Deb, K., Chen, Y. P., Auger, A., \& Tiwari, S. (2005). Problem definitions and evaluation criteria for the CEC 2005 special session on real-parameter optimization. KanGAL report, 2005005.

Tizhoosh, H.R. (2005). Opposition-based learning: a new scheme for machine intelligence. International conference on computational intelligence for modelling control and automation; Austria. p. 695-701.

Van den Bergh, F., \& Engelbrecht, A. P. (2004). A cooperative approach to particle swarm optimization. Evolutionary Computation, IEEE Transactions on,8(3), 225-239.

Zaharie, D. (2009). Influence of crossover on the behavior of differential evolution algorithms. Applied Soft Computing, 9(3), 1126-1138.

Zolfaghari, A. R., Heath, A. C., \& McCombie, P. F. (2005). Simple genetic algorithm search for critical non-circular failure surface in slope stability analysis. Computers and Geotechnics, 32(3), 139-152.

Rao, R., \& Patel, V. (2013). Comparative performance of an elitist teaching-learning-based optimization algorithm for solving unconstrained optimization problems. International Journal of Industrial Engineering Computations, 4(1), 29-50.

Rao, R., \& Patel, V. (2012). An elitist teaching-learning-based optimization algorithm for solving complex constrained optimization problems. International Journal of Industrial Engineering Computations, 3(4), 535-560.

Rao, R. V., Savsani, V. J., \& Vakharia, D. P. (2011). Teaching-learning-based optimization: a novel method for constrained mechanical design optimization problems. Computer-Aided Design, 43(3), 303-315. 
Appendix-1

\begin{tabular}{|c|c|c|c|c|c|}
\hline $\mathrm{F}$ & Function & Formulation & $\mathrm{D}$ & $\begin{array}{l}\text { Search } \\
\text { Space }\end{array}$ & $f_{\min }$ \\
\hline 1 & Sphere & $f(x)=\sum_{i=1}^{D} x_{i}^{2}$ & 50 & {$[-100,100]$} & 0 \\
\hline 2 & $\begin{array}{l}\text { Schwefel2. } \\
22\end{array}$ & $f(x)=\sum^{D}\left|x_{i}\right|+\prod^{D}\left|x_{i}\right|$ & 50 & {$[-10,10]$} & 0 \\
\hline 3 & $\begin{array}{l}\text { Schwefel1. } \\
2\end{array}$ & $f(x)=\sum^{D} \sum^{i} x_{i}^{2}$ & 50 & {$[-100,100]$} & 0 \\
\hline 4 & $\begin{array}{l}\text { Schwefel2. } \\
21\end{array}$ & $f(x)=\max \left\{\left|x_{i}\right|, 1 \leq i \leq D\right\}$ & 50 & {$[-100,100]$} & 0 \\
\hline 5 & Rosenbrock & $f(x)=\sum_{i=1}^{D}\left[100\left(x_{i+1}-x_{i}^{2}\right)^{2}+\left(x_{i}-1\right)^{2}\right]$ & 50 & {$[-30,30]$} & 0 \\
\hline 6 & Step & $f(x)=\sum_{i=1}^{D}\left(x_{i}+0.5\right)^{2}$ & 50 & {$[-100,100]$} & 0 \\
\hline 7 & Quartic & $f(x)=\sum_{i}^{D} i x_{i}^{4}+\operatorname{random}(0,1)$ & 50 & {$[-1.28,1.28]$} & 0 \\
\hline 8 & Schwefel & $f(x)=-\sum x_{i} \sin \left(\sqrt{\left|x_{i}\right|}\right)$ & 50 & {$[-500,500]$} & $\begin{array}{l}- \\
418.9828872724338^{*} \\
\text { D }\end{array}$ \\
\hline 9 & Rastrigin & $f(x)=10 D+\sum_{i=1}^{D}\left[x_{i}^{2}-10 \cos \left(2 \pi x_{i}\right)\right]$ & 50 & {$[-5.12,5.12]$} & 0 \\
\hline 10 & Ackley & $f(x)=20+e-20 e^{\frac{1}{D}\left(\sqrt{\frac{1}{D} \sum_{i=1}^{D} x_{i}^{2}}\right)}-e^{\frac{1}{D}\left(\sum \cos \left(2 \pi x_{i}\right)\right)}$ & 50 & {$[-32,32]$} & 0 \\
\hline 11 & Griewank & $f(x)=\sum_{i=1}^{D} \frac{x_{i}^{2}}{4000}-\prod_{i=1}^{D} \cos \left(\frac{x_{i}}{\sqrt{i}}\right)-1$ & 50 & {$[-600,600]$} & 0 \\
\hline 12 & Penalized1 & $f(x)=\frac{\pi}{D}\left\{\begin{array}{l}10 \sin ^{2}\left(\pi y_{i}\right)+\sum_{i=1}^{D-1}\left(y_{i}-1\right)^{2}\left[1+10 \sin ^{2}\right. \\
\left.+\left(y_{D}-1\right)^{2}\right]\end{array}\right.$ & 50 & {$[-50,50]$} & 0 \\
\hline & & $+\sum_{i=1}^{D} u\left(x_{i}, 10,100,4\right)$ & & & \\
\hline & & $\begin{array}{l}\text { Where } y_{i}=1+\frac{1}{4}\left(x_{i}+1\right), \\
u\left(x_{i}, a, k, m\right)=\left\{\begin{array}{cc}k\left(x_{i}-a\right)^{m} & x_{i}>a \\
0 & -a<x_{i}<a \\
k\left(-x_{i}-a\right)^{m} & x_{i}<a\end{array}\right.\end{array}$ & & & \\
\hline
\end{tabular}

13 Penalized2

$$
\begin{aligned}
& f(x)=0.1\left\{\begin{array}{l}
10 \sin ^{2}\left(\pi x_{i}\right)+\sum_{i=1}^{D-1}\left(x_{i}-1\right)^{2}\left[1+10 \sin ^{2} 50 \quad[-50,50]\right. \\
\left.+\left(x_{D}-1\right)^{2}\left[1+\sin ^{2}\left(2 \pi x_{D}\right)\right]\right]
\end{array}\right. \\
& +\sum_{i=1}^{D} u\left(x_{i}, 5,100,4\right)
\end{aligned}
$$


14 Foxholes

$$
f(x)=\left[\frac{1}{500}+\sum_{j=1}^{25} \frac{1}{j+\sum_{i=1}^{2}\left(x_{i}-a_{i j}\right)}\right]^{-1}
$$

15 Kowalik

$$
f(x)=\sum_{i=1}^{11}\left[a_{i}-\frac{x_{1}\left(b_{i}^{2}+b_{i} x_{2}\right)}{b_{i}{ }^{2}-b_{i} x_{3}-x_{4}}\right]^{2}
$$

16 Six Hump

$$
f(x)=4 x_{1}^{2}-2.1 x_{1}^{4}+\frac{1}{3} x_{1}^{6}+x_{1} x_{2}-4 x_{2}^{2}+4 x_{2}^{2}
$$

17 Branin

$$
f(x)=\left(x_{2}^{2}-\frac{5.1}{4 \pi^{2}} x_{1}^{2}+\frac{5}{\pi} x_{1}-6\right)^{2}+10\left(1-\frac{1}{8 \pi}\right)^{2} \quad \begin{aligned}
& {[-5,10] \text { and }} \\
& {[0,15]}
\end{aligned}
$$

18 Goldstein

$$
\begin{aligned}
& f(x)=\left[1+\left(x_{1}+x_{2}+1\right)^{2}\left(10-14 x_{1}+3 x_{1}^{2}\right.\right. \\
& \left.\left.-14 x_{2}+6 x_{1} x_{2}+3 x_{2}^{2}\right)\right] \times\left[30+\left(2 x_{1}-3 x_{2}^{2}\right)(18-\right. \\
& \left.\left.+12 x_{1}^{2}+48 x_{2}-36 x_{1} x_{2}+27 x_{2}^{2}\right)\right]
\end{aligned}
$$$$
2 \quad[-2,2]
$$

19 Shekel5

$$
f(x)=-\sum_{i=1}^{5}\left[\left(x-a_{i}\right)\left(x-a_{i}\right)^{T}+c_{i}\right]^{-1}
$$

$20 \quad$ Shekel7

$$
f(x)=-\sum_{i=1}^{7}\left[\left(x-a_{i}\right)\left(x-a_{i}\right)^{T}+c_{i}\right]^{-1}
$$

21 Shekel10

$$
f(x)=-\sum_{i=1}^{10}\left[\left(x-a_{i}\right)\left(x-a_{i}\right)^{T}+c_{i}\right]^{-1}
$$

